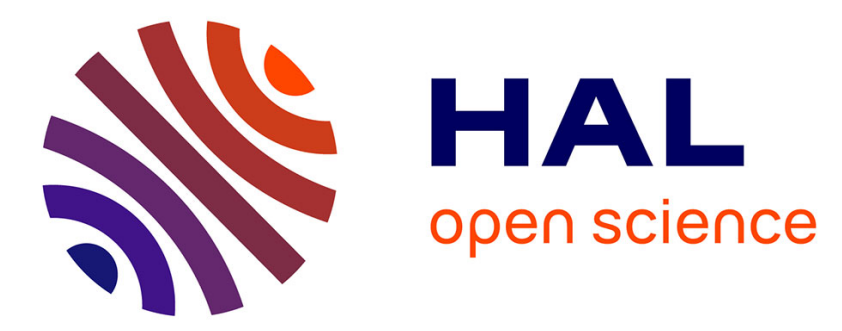

\title{
Effects of biomolecules on the electrokinetics of colloidal nanoparticles in liquid suspension
}

\author{
Clyde Midelet, J.-Y. Lin, Sung Tsang, Chen-Li Sun, Johanna Midelet, \\ Antonios G. Kanaras, Bruno Le Pioufle, Olivier Français, Martinus H. V. H V \\ Werts
}

\section{To cite this version:}

Clyde Midelet, J.-Y. Lin, Sung Tsang, Chen-Li Sun, Johanna Midelet, et al.. Effects of biomolecules on the electrokinetics of colloidal nanoparticles in liquid suspension. SPIE BIOS, Jan 2017, San Francisco, CA, United States. pp.100780T, 10.1117/12.2252470 . hal-01501667

\section{HAL Id: hal-01501667 https://hal.science/hal-01501667}

Submitted on 18 Nov 2017

HAL is a multi-disciplinary open access archive for the deposit and dissemination of scientific research documents, whether they are published or not. The documents may come from teaching and research institutions in France or abroad, or from public or private research centers.
L'archive ouverte pluridisciplinaire HAL, est destinée au dépôt et à la diffusion de documents scientifiques de niveau recherche, publiés ou non, émanant des établissements d'enseignement et de recherche français ou étrangers, des laboratoires publics ou privés. 


\title{
Effects of biomolecules on the electrokinetics of colloidal nanoparticles in liquid suspension (invited paper)
}

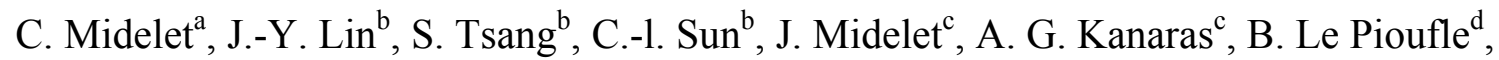 \\ O. Français ${ }^{\mathrm{e}}, \mathrm{M}$. H. V. Werts*a \\ ${ }^{a}$ SATIE (UMR 8029), Ecole normale supérieure de Rennes, CNRS, Campus de Ker Lann, F-35170 \\ Bruz, France; ${ }^{b}$ Department of Mechanical Engineering, National Taiwan University, Taipei, \\ Taiwan; ${ }^{c}$ Department of Physics and Astronomy, University of Southampton, Southampton, \\ England; ${ }^{\mathrm{d}}$ SATIE (UMR 8029), Ecole normale supérieure Paris-Saclay, F-94235 Cachan, France F- \\ 94235 Cachan, France; ${ }^{e}$ ESIEE-Paris, ESYCOM, University Paris-Est, F-93160 Noisy Le Grand, \\ France.
}

\begin{abstract}
Electric fields can induce various types of motion in liquid suspensions of colloidal nanoparticles. These electrokinetic phenomena depend on the parameters of the electric field (frequency, amplitude, 3D topology), the particles (size, shape, composition) and the suspending liquid (polarizability, ionic strength, $\mathrm{pH}$ ). In particular, the dielectrophoretic force on submicron colloidal particles is dependent on the properties of the electric double layer (the "ion cloud") around these particles. This dependence provides a mechanism for detecting and quantifying interactions between biomolecules and these nanoparticles, which can be combined with optical and spectroscopic measurements. Here, we report on functionalized plasmonic nanoparticles that are tracked inside microfluidic systems by dark-field video-microscopy. A high-gradient $\mathrm{AC}$ electric field is set up using transparent micro-electrodes. Electrohydrodynamic motion of the entire fluid and dielectrophoretic trapping of individual particles can be analyzed quantitatively by numerical methods. By switching the electric field synchronously with the video acquisition, the effect of biomolecules on the electrokinetic trapping can be quantified. The electromicrofluidic devices allow also for rapid measurement of diffusion coefficients.
\end{abstract}

Keywords: electrokinetics, dielectrophoresis, microfluidics, resonant light scattering, video microscopy, colloidal suspensions, nanoparticles, plasmonic nanoparticles

\section{INTRODUCTION}

The combination of microfluidics and colloidal suspensions of inorganic nanocrystals is of interest for a number of reasons. It can lead to the development of new analytical detection strategies for in vitro diagnostics and environmental monitoring, ${ }^{1-2}$ where the nanocrystals interact with the analytes and the microfluidic device provides the infrastructure for sample processing and read-out. Microfluidics can also contribute to precise control over nanoparticle synthesis and purification ${ }^{3-4}$, with the complementary attraction of providing a continuous flow process. A further interest lies in the fact that microfluidic platforms can be used for characterization of nanoparticles directly in their native colloidal suspension. This is illustrated for instance by the microfluidic measurement of diffusion coefficients. ${ }^{5-6}$ Integration of micro-electrodes into the microfluidic device gives access to measurement of electrokinetic parameters of the colloidal suspensions, which depend on the surface state of the colloids. ${ }^{7}$ This may for example be useful for monitoring ligand exchange $^{8}$ and desorption ${ }^{9}$ at the nanoparticle surface.

For electrokinetic studies in microfluidic systems, the use of alternating (AC) electric fields is preferred in order to avoid electrochemical reactions at the electrodes (water electrolysis, redox effects, change of ph, deterioration of the electrodes). ${ }^{10}$ The electrokinetic phenomena then observed can be classified into electrohydrodynamics (EHD), where the entire fluid mass is put into motion by the AC field, and dielectrophoresis (DEP), where the individual particles are subject to a force induced by the AC electric field topology.

*martinus.werts@ens-rennes.fr; phone+332990593 47; fax +33299059328 
It is well known that the time-averaged dielectrophoretic force on spherical particles of radius $R_{\mathrm{NP}}$ in a medium with permittivity $\varepsilon_{m}$ is given by ${ }^{11}$

$$
\boldsymbol{F}_{\mathrm{DEP}}=2 \pi R_{\mathrm{NP}}^{3} \varepsilon_{m} K_{C M} \boldsymbol{\nabla} E_{\mathrm{RMS}}^{2}
$$

The force follows the square of the gradient of the time-averaged magnitude $\left(E_{\mathrm{RMS}}\right)$ of the electric field, and furthermore depends on $K_{C M}$ which is the real part of the Clausius-Mossotti factor, $K_{\mathrm{CM}}=\operatorname{Re}\left(f_{\mathrm{CM}}\right){ }^{12}$ For nanoparticles, $K_{C M}$ mostly depends on the (frequency-dependent) electrical properties of the nanoparticle surface and the surrounding electric double layer. It can range from +1 to -0.5 , and its sign determines the direction of the DEP force (positive DEP if $K_{C M}>\mathbf{0}$, repulsive in the other case, negative DEP).

The micro-electrodes for DEP should thus be designed to provide strong field gradients. A wide variety of electrode architectures for dielectrophoresis in microfluidic systems has been proposed over the years. ${ }^{73}$ Three-dimensional electrode architectures are particularly attractive for manipulating colloidal particles in suspension, because of the possibility of trapping the particles in the middle of a microfluidic channel, in the bulk fluid, away from the bottom and top walls. ${ }^{14}$ However, the fabrication of such $3 \mathrm{D}$ architecture is more complicated, and for many analytical applications flat 2D electrode structures may be sufficient.

For our studies on the electrokinetics of gold nanoparticle suspensions we chose two-dimensional curved thin-film electrodes with sharp tips (see Figure 1). This architecture was developed recently ${ }^{15}$ and used successfully with submicron particles. ${ }^{16}$ The region of high field gradient is strongly localized near the tips, defining a clear 'hot spot' for dielectrophoretic capturing of nanoparticles. Moreover, the curved design facilitates the combination of microfluidic flow and electrokinetics for microscale manipulation of nanoparticles by allowing a dense array of electrode pairs to be integrated.
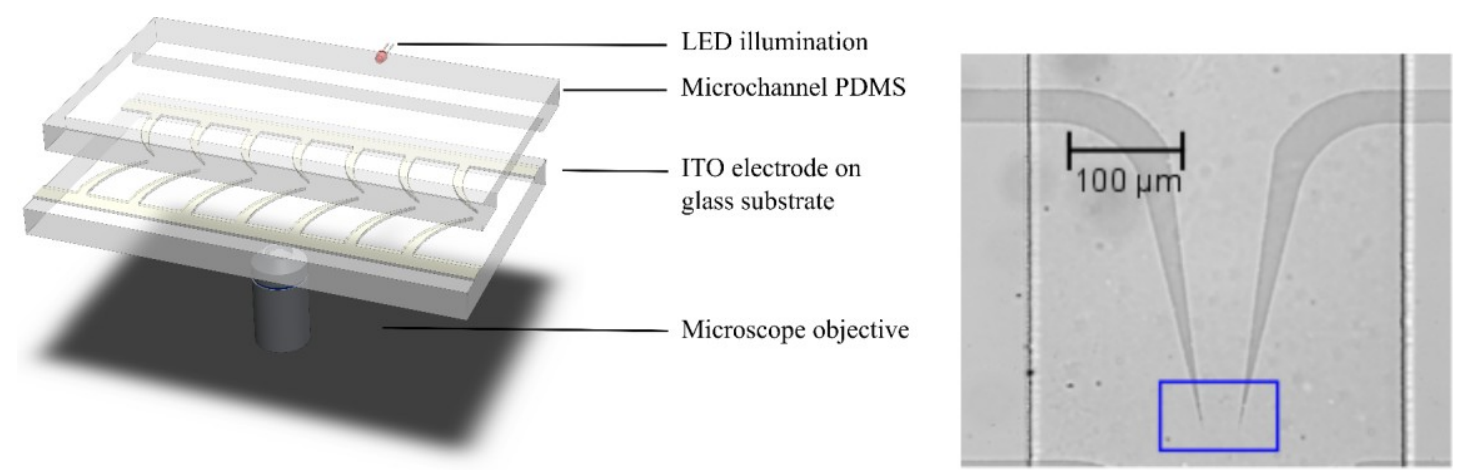

Figure 1. (Left) Schematic representation of a transparent (PDMS on glass electrode substrate) microfluidic device with LEDs for dark field illumination. The LED light is side-coupled into the PDMS slab which then acts as a light guide. Scattered light, as well as fluorescence are observed through the glass electrode substrate using a microscope objective. (Right). Micrograph (bright field) of patterned ITO microelectrodes in a PDMS microchannel. Contrast has been enhanced to bring out the features of the ITO electrodes. The blue rectangle represents a typical 'region of interest' used for dark-field videomicroscopic recording of the electrokinetic response of gold nanoparticles.

In previous work, ${ }^{1}$ the electrodes were made of $200 \mathrm{~nm}$ thick gold (with $30 \mathrm{~nm} \mathrm{Ti}$ adhesion layer), which is not transparent and therefore does not enable imaging those particles that are moving behind the electrode tip. Here we use optically transparent electrodes microfabricated in indium-tin oxide (ITO), an optically transparent conducting material. We present new methodology for real-time monitoring and quantitative measurement of dielectrophoretic capturing of functionalized gold colloids, and demonstrate how this can be used to study the effect of biomolecules on this process. As illustrative molecules of biological origin, lipoic acid and bovine serum albumin were chosen. Both have already been demonstrated to interact with gold nanoparticles ${ }^{17-18-19}$ and change physicochemical behavior of these particles. 


\section{MATERIALS AND METHODS}

\subsection{Gold nanoparticles and biomolecules functionalization}

Aqueous colloidal suspensions of gold nanospheres of $150 \mathrm{~nm}$ diameter were obtained from a commercial source (BBInternational, Cardiff, Wales, UK). Spherical gold nanoparticles of $70 \mathrm{~nm}$ diameter (referred to as "Aug6") were synthesised according to a recently published seeded-growth method developed by Puntes and co-workers. ${ }^{20}$ First, $10 \mathrm{~nm}$ AuNPs seeds were synthesised by adding a gold salt solution $(1 \mathrm{~mL}, 25 \mathrm{mM})$ into a boiling solution of trisodium citrate $(150 \mathrm{~mL}, 2.2 \mathrm{mM})$. After 10 minutes the temperature was reduced to $90^{\circ} \mathrm{C}$. The addition of gold salt solution $(1 \mathrm{~mL}, 25$ $\mathrm{mL}$ ) follow by 30 minutes, reaction time was repeated twice to achieved the first growth step (Aug0). The solution was then diluted by extracting $55 \mathrm{ml}$ and adding a solution of sodium citrate $(55 \mathrm{~mL}, 2.2 \mathrm{mM})$. Six other sequential growth steps were performed yielding to particles of $70.8 \pm 8.7 \mathrm{~nm}$. Transmission electronic microscopy (TEM) was used to characterise the sample.
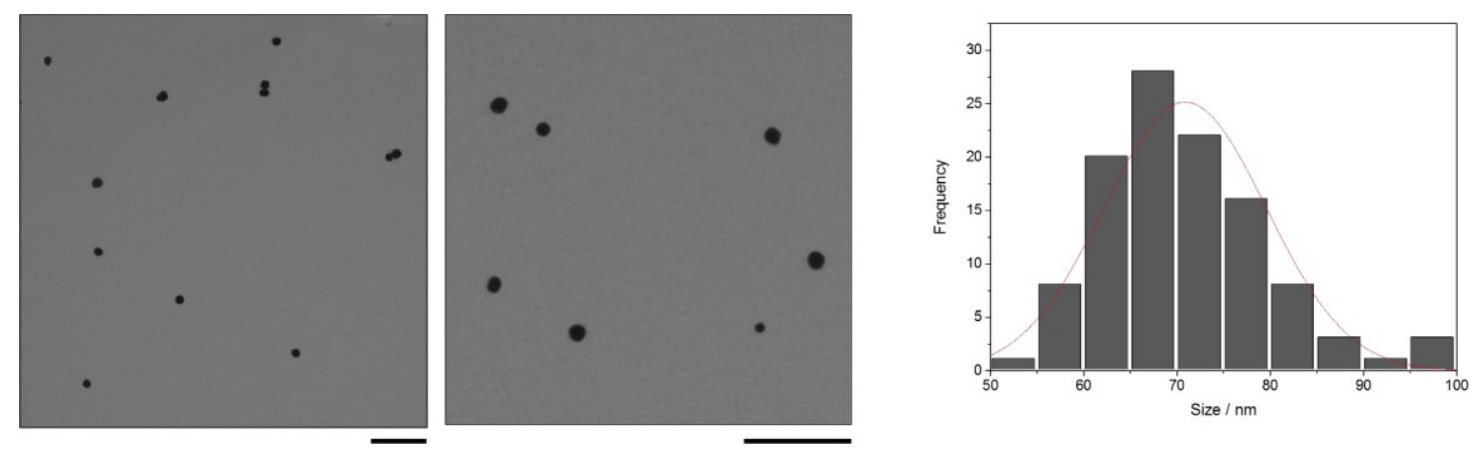

Figure 2. (Left) TEM micrographs of "Aug6" $70 \mathrm{~nm}$ gold nanospheres. Scale bars are $500 \mathrm{~nm}$. (Right) Histogram of the size distribution for Aug6.

Nanosphere concentrations were determined by UV-visible spectroscopy using extinction coefficients obtained from the specified particle diameters through known relations based on Mie theory ${ }^{21}$ Functionalization of the colloidal particles was achieved by mixing them with aqueous solutions of biomolecules: either racemic $( \pm)$ - $\alpha$-lipoic acid (LA, Sigma, 0.2 $\mathrm{mM}$ ) or bovine serum albumin (BSA, Acros Organics, Belgium, $100 \mathrm{nM}$ ) in $1 \mathrm{mM}$ aqueous NaOH. Typically, the samples were centrifuged (Mikro 220R, Hettich) for $30 \mathrm{~min}$ at $450 \mathrm{x} g$ or $600 \mathrm{x} g$ for Au150 and Aug6, respectively. Then the supernatant was removed and replaced ( $92 \%$ of the volume) by the solutions containing the biomolecules. This centrifugation-redispersion was repeated three times, to obtain functionalised gold particles: Au150-LA, Aug6-LA and Au150-BSA.

\subsection{Optical spectroscopy}

Optical spectroscopy was carried out at ambient temperature on air-equilibrated samples contained in standard $1 \mathrm{~cm}$ plastic (PMMA) fluorescence cuvettes. UV-visible extinction spectra were measured using an optical fiber-based system (Ocean Optics) incorporating a USB4000-VIS-NIR CCD spectrometer and a LS-1 tungsten halogen light source. For resonant light scattering (RLS) measurements ${ }^{17-21}$ the samples were diluted to an optical density well below 0.05 and illuminated with white light (Ocean Optics LS-1). The scattered light was collected at a fixed angle of $90^{\circ}$, and analysed using a CCD spectrograph (Ocean Optics QE65000). Although measurement at right angles does not account for the angular distribution of light scattering (which is of particular importance for gold nanoparticles of diameters $>80 \mathrm{~nm}$ ) the error in the measurement of the scattering cross section is expected to be below $10 \%$ for 150 nm diameter gold nanospheres. ${ }^{22}$ A water background was subtracted from all recorded spectra, and the spectra were then corrected using Ludox as the perfect Rayleigh scatterer. The Ludox sample consisted of the supernatant of a Ludox SM30 (Aldrich) suspension centrifuged for $1 \mathrm{~h}$ at $9700 \mathrm{x}$ g (9990 RPM), diluted 200 times in $0.05 \mathrm{M} \mathrm{NaCl}$. Three Ludox reference samples were characterized using their $p_{0}$ value, by fitting $\operatorname{OD}_{\text {Ludox }}(\lambda)=p_{0} \lambda^{-4}+p_{1}$ to the experimental Ludox extinction spectrum. The baseline value $p_{1}$ must be very close to zero. It it was verified that all three Ludox samples gave identical 
raw scattering spectra, and the average of their $p_{0}$ values was used. The corrected light scattering spectra were obtained according to Eqn (1) by calibration of the spectrum against the Ludox reference, taking also into account the coefficient $p_{0}$. The subscript 'LILS' signifies 'Ludox-independent light scattering', meaning that the spectrum has been corrected using the Ludox reference and that the density of the Ludox ( $p_{0}$ parameter) has been taken into account. We do this to avoid confusion with light scattering spectra which have been corrected against Ludox, but without accounting for the density of the Ludox.

$$
I_{L I L S}=p_{0} \lambda^{-4} \frac{I_{\text {raw }}(\lambda)}{I_{\text {Ludox }}(\lambda)}
$$

To compare the light scattering spectra of different samples, normalization with respect to the concentration of particles is necessary. Here we achieve this by using the maximum value of the extinction spectrum of the RLS sample. Eqn (2)

$$
I_{L I L S}^{\text {norm }}=\frac{I_{L I L S}(\lambda)}{O D\left(\lambda_{\max }\right)}
$$

\subsection{Electromicrofluidic device}

The electrodes were patterned on indium-tin oxide thin film (ITO, $80 \mathrm{~nm}$ ) covered soda-lime glass slides $(1.1 \mathrm{~mm}$ thick, Solems S.A., Palaiseau, France). First, the slides were rinsed with acetone, isopropanol and water, and dried. Positive photoresist "S1805" (Rohm and Haas, USA) was then deposited onto the substrate by spin-coating for $30 \mathrm{~s}$ at $1000 \mathrm{rpm}$ with $100 \mathrm{rpm} / \mathrm{s}$ acceleration. The thickness of photoresist obtained was $800 \mathrm{~nm}$. Then, the photoresist was exposed to UV (i-line $365 \mathrm{~nm} ; 180 \mathrm{~mJ} / \mathrm{cm}^{2}$ dose) on an MJB4 (Süss MicroTec, Germany) mask aligner using a quartz mask (JD Photo-Tools, UK) with the electrode design. After development ( $1 \mathrm{~min}$. in "351 developer", Rohm and Haas, diluted in deionized water 1 :4), wet etching of the ITO layer was achieved using $\mathrm{HCl}$ in water $\left(1 \mathrm{~mol}^{-1}\right)$ during 5 min at $40^{\circ} \mathrm{C}$ (etching rate $4 \mathrm{~nm} \mathrm{~s}^{-1}$ ). A final solvent cleaning (acetone, isopropanol, water, drying) was done in order to remove the photoresist. Before each use the ITO electrode substrates were cleaned using a low-pressure air plasma for 5 minutes (Harrick Plasma Cleaner/Sterilizer PDC-002, USA). They were then treated with diluted gold etch solution (150 mM potassium iodide, $25 \mathrm{mM} \mathrm{I}_{2}$ in water) during $4 \mathrm{~min}$ at room temperature $\left(19^{\circ} \mathrm{C}\right)$, thoroughly rinsed with deionized water and dried under a filtered nitrogen stream.

The microfluidic circuits were molded into an optically transparent silicone elastomer. For fabrication of the mold, a 40 $\mu \mathrm{m}$ thick SU-8 layer (Microchem SU-8 3050) is spin-coated on top of a 4" silicon wafer. A Süss-MicroTec MJB4 mask aligner is used for the photolithography step. Polydimethylsiloxane (PDMS Sylgard 184 - Dow Corning) was chosen as the material for the microfluidic chip. A 10:1 mix of PDMS and its curing agent is degassed under reduced pressure (2 hours) and then poured on top of the SU-8 mold in a Petri dish. The Petri dish containing the mold and the PDMS is once again degassed, and cured for one hour at $70^{\circ} \mathrm{C}$ before the PDMS is peeled off the mold. Microfluidic chips are then cut, and inlets/outlets are punched.

The electromicrofluidic device was assembled by positioning the PDMS microfluidic circuitry on top of the ITO/glass electrode substrate. Prior to assembly, the bottom of the PDMS circuit was wetted with a small quantity of isopropanol or ethanol which enables free movement of the PDMS on top of the substrate. Positioning was then achieved manually, monitoring alignment of the microfluidic channel and the electrode structure using an optical microscope. Once aligned, the device was left to dry, and microfluidic tubing was connected. The microfluidic circuit was operated using pressuredriven flow. Fine control of the pressure at the exit of the microfluidic circuit (and hence the pressure difference between entrance and exit) was achieved using a screw syringe (total volume $60 \mathrm{~mL}$, Jeulin, Evreux, France) filled with air. Air pressure was monitored using a digital pressure meter (Jeulin).

\subsection{Dark-field videomicroscopy}

The microfluidic device was observed on an Olympus IX71 inverted microscope, using low and moderate magnification objectives $(2 \mathrm{x}, 4 \mathrm{x}, 10 \mathrm{x}, 40 \mathrm{x}$, N.A. $=0.06,0.1,0.3$ and 0.6 respectively). Electrokinetic videos were obtained using the 40x objective (depth-of-field approx. $2 \mu \mathrm{m}$ ) with the focal plane $2 \mu \mathrm{m}$ above the electrodes. For dark-field illumination, ${ }^{6-}$

10-12, LEDs (3 mm diameter, typical operation current $20 \mathrm{~mA})$ of the desired wavelength $(620 \mathrm{~nm}$ or $525 \mathrm{~nm})$ were mounted in a $5 \times 2$ insulation-displacement connector (IDC) on a 10-wire ribbon cable. The connector was positioned in such a way that allowed the LED to illuminate the side of the PDMS device. LED power was supplied by 4 rechargeable $1.2 \mathrm{~V}$ batteries $(\mathrm{NiMH})$. 
The videomicroscopy experiment was controlled using Micro-Manager software (Version 1.4) ${ }^{23-24}$ running under the Windows 7 (64-bit) operating system. Images were acquired using a Zyla 5.5 s-CMOS camera (Andor technology, UK). LED illumination and electric field switching were controlled using home-built electronics based on an Arduino Uno board and a ULN2803A Darlington transistor array for switching LED power. The Arduino Uno board communicates directly with the Micro-Manager software using a modified version of the custom Micro-Manager Arduino firmware. ${ }^{25}$ Electric field was generated by applying the output of function generator (Centrad GF266, ELC, France) to a selected pair of ITO electrodes on the substrate. The function generator generates a sine wave of specified frequency (in this work $1 \mathrm{MHz}$ ) and amplitude (up to $15 \mathrm{~V}_{\mathrm{pp}}$ ). The AC electric field was switched on and off ('amplitude modulated') via the Arduino electronics, which also switches the LED illumination. This finally allows for a fully synchronized acquisition cycle, during which the electric field is switched on and off at precisely defined time intervals.

Typically, videos were acquired for $2 \ldots 10$ minutes at 50 frames per second, cycling the electric field between off and on with a period of $15 \mathrm{~s}$ (11 s 'OFF', $4 \mathrm{~s}$ 'ON'). Synchronization of the electric field switching with video acquisition was further ensured by briefly blinking the LED illumination $(10 \mathrm{~ms})$ when switching the electric field from 'ON' to 'OFF'. The timing of the blinking (and the electric field switching) was found to be precise to a single frame (better than $20 \mathrm{~ms}$ ).

For analysis of the dielectrophoretic capturing of the gold nanoparticles, the videomicroscopy sequences were processed using Python 3.5.2, with the 'numpy', 'scipy' and 'matplotlib' libraries (Anaconda 4.1.1, 64-bit, Continuum Analytics, USA $)^{26-27-28}$. The Micro-Manager video files are in OME-TIFF format and read into computer memory using the 'tifffile.py' module ${ }^{29}$ prior to processing.

For analysis of the electrohydrodynamic flow characteristics in the microfluidic system, microscale particle image velocimetry ( $\mu$-PIV) is employed. ${ }^{30}$ The acquired images are analyzed by PIVLab ${ }^{31}$ which implements cross-correlation to determine the displacement of glowing nanoparticles between two consecutive frames. The actual field of interest is subdivided into interrogation areas of $16 \times 16$ pixels $\left(2.6 \times 2.6 \mu \mathrm{m}^{2}\right)$. Each interrogation cell contains approximately $4-5$ nanoparticles. The LED synchronization blink is employed to assign the reference phase so that we are able to obtain the velocity field and flow pattern at a given phase by averaging over several subsequent electric-field switching cycles. For gold nanoparticles with a mean diameter of $150 \mathrm{~nm}$, the corresponding hydrodynamic relaxation time is $27.1 \mathrm{~ns}$ in the solution, which is much smaller than the time interval between frames.

\section{RESULTS AND DISCUSSION}

\subsection{Optical properties of gold nanoparticles}

The choice of LED wavelength for dark-field observation in the microsystem is based on an optical spectroscopic characterization of the gold nanoparticle suspensions. The extinction and scattering spectra of Au150-LA, Aug6-LA and Au150-BSA agree well with the expectations obtained from Mie theory, which demonstrates their chemical integrity. The $150 \mathrm{~nm}$ gold nanoparticles display a very broad plasmon resonance in their extinction and scattering spectra (Figure 2 ), which is red-shifted with respect to the better-known $520 \ldots 540 \mathrm{~nm}$ plasmon resonance for smaller $(10 \ldots 80 \mathrm{~nm})$ gold nanospheres. Retardation effects lead to the break-down of the simple dipolar approximation for the localized plasmon resonance (and hence the appearance of a red-shifted resonance), but full Mie theory still reproduces the optical spectra of these large particles.
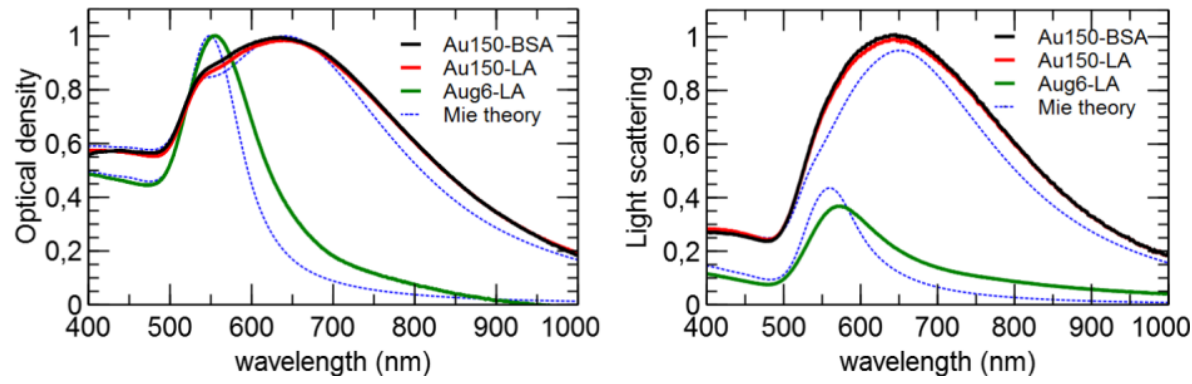

Figure 3. Extinction (left) and light scattering (right) spectra of $150 \mathrm{~nm}$ and $70 \mathrm{~nm}$ gold nanoparticles in water $(1 \mathrm{mM} \mathrm{NaOH})$, coated with lipoate (LA) or bovine serum albumin (BSA) 
An important observation is that changing the functionalization of the nanoparticle from lipoate to BSA only induces a very minor change in the optical properties (Table 1). Finally, for the Au150 particles a red LED $\left(\lambda_{\text {em }}=625 \mathrm{~nm}\right)$ was used for dark-field illumination, and for the Aug6 particles a green LED $\left(\lambda_{\mathrm{em}}=525 \mathrm{~nm}\right)$.

\begin{tabular}{ccccc}
\hline Particles & $\mathrm{d}(\mathrm{nm})$ & Ligands & $\lambda_{\max }$ ext. $(\mathrm{nm})$ & $\lambda_{\max }$ RLS $(\mathrm{nm})$ \\
\hline Au150-LA & \multirow{2}{*}{150} & LA & 641.1 & 642.5 \\
Au150-BSA & & BSA & 633.6 & 642.9 \\
Aug6-LA & 70 & LA & 555.2 & 572.6 \\
\hline
\end{tabular}

Table 1. Position of the maximum plasmon resonance $\lambda_{\max }$ in the extinction spectra and light scattering spectra of gold nanoparticles in water $(1 \mathrm{mM} \mathrm{NaOH})$

\subsection{Video microscopy of electromicrofluidic behavior of functionalized colloidal gold suspensions}

With the side-LED-illuminated dark field, the individual gold nanoparticles in the microfluidic device are clearly visible by eye through the microscope using 10x and 40x objectives, and are recognizable via their rapid and incessant Brownian motion. Video sequences are recorded. In the present work, we are not concerned with tracking individual particles, but with their ensemble behavior, i.e. how their concentration evolves in space and time. Taking this viewpoint, we can record video sequences over several cycles of switching on and off the electric field, and then average the video frames.

We established previously that at an electric field frequency of $1 \mathrm{MHz}$, dielectrophoretic capturing of $150 \mathrm{~nm}$ gold particles strongly dominates other effects, ${ }^{1}$ and the study reported here exclusively uses an AC field at 1 MHz. However, in the case of Au150-LA particles in $1 \mathrm{mM} \mathrm{NaOH}$, electrohydrodynamic motion of the entire fluid was observed at higher field amplitudes $\left(10 \mathrm{~V}_{\mathrm{pp}}, 15 \mathrm{~V}_{\mathrm{pp}}\right)$. We tentatively attribute this to the electrothermal effect, ${ }^{7}$ in which the small electrical currents in the fluid (conductivity of the solution: $2.5 \times 10^{-2} \mathrm{~S} \mathrm{~m}^{-1}$ ) set up a thermal gradient to which is associated a gradient of the electric permittivity. The electric permittivity gradient leads to a body force on the fluid mass in the applied electric field, and sets the entire fluid in motion.

Figure 4 shows a typical $\mu$ PIV velocity map of the induced fluid motion. Herein, the averaged result of $t_{\mathrm{ON}}=1 \ldots 2 \mathrm{~s}$ (i.e. $\mathrm{t}=-3 \ldots-2 \mathrm{~s}$ before switching OFF) is shown because the movement of nanoparticles exhibits a transient response during the first second after switching on the electric field. As seen in Figure 4, the asymptotic trajectories form two positive bifurcation lines on top of the electrodes and a negative bifurcation line laying in between the electrodes. The flow pattern suggests that two counter-rotating vortex pairs are induced and the four vortex tubes are aligned along the two electrodes. As a result, the rotating motion of nanoparticles has significant out-of-plane velocity component which leads to the decrease in velocity magnitude at the edges of the vortices. This is ascribed to the constraint of our $\mu$ PIV analysis which evaluates the projection of three-dimensional movement of nanoparticles onto the two-dimensional image plane. Despite this limitation, the maximal velocity somehow represents the angular velocity at which nanoparticles circle around the vortex axis. From Figure 4, this velocity is about $13.2 \mu \mathrm{m} / \mathrm{s}$ for $\mathrm{V}_{\mathrm{pp}}=15 \mathrm{~V}$. For $\mathrm{V}_{\mathrm{pp}}=10 \mathrm{~V}$ and $5 \mathrm{~V}$, weaker rotation is observed and the maximal velocity reduces to $1.9 \mu \mathrm{m} / \mathrm{s}$ and $0.5 \mu \mathrm{m} / \mathrm{s}$ respectively. This latter value falls below the uncertainty limit of the velocity determination. 

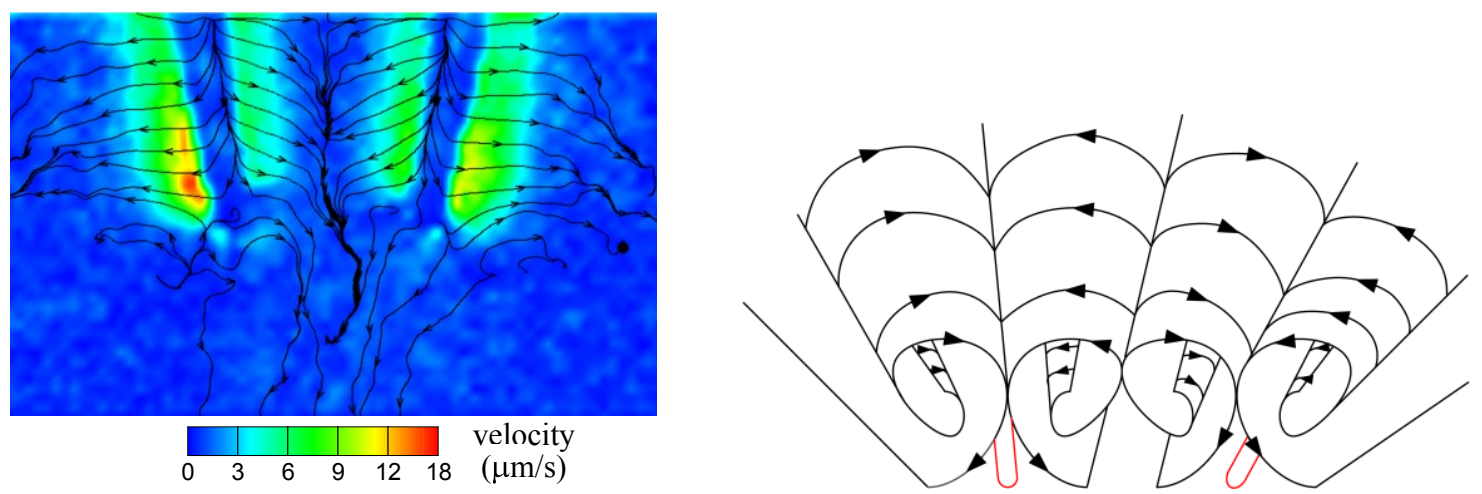

Figure 4. A typical $\mu \mathrm{PIV}$ velocity map of the induced flow field averaged over $\mathrm{t}=-3 \ldots-2 \mathrm{~s}$ (i.e. $1 \ldots 2 \mathrm{~s}$ after switching the $\mathrm{E}$ field on), $\mathrm{V}_{\mathrm{pp}}=15 \mathrm{~V}, 1 \mathrm{MHz}$. The focal observation plane was just $2 \mu \mathrm{m}$ above the electrodes Right: Sketch of proposed fluid motion

The observed electrohydrodynamic (EHD) motion, tentatively attributed to the electrothermal effect, should be minimized in order to study 'pure' dielectrophoresis of the gold nanoparticles. The above result suggests that EHD is minimized for field amplitudes below $10 \mathrm{~V}_{\mathrm{pp}}$. The dielectrophoretic capturing of the nanoparticles was monitored by recording videos of the light scattering intensity distribution, while switching the electric field on and off in a periodic manner.

Each pixel of the video image has an intensity $I_{\text {video }}$ that receives contributions from detector dark current $I_{d a r k}$, stray light $I_{\text {stray, }}$ and the detected scattered light from the nanoparticles.

$$
I_{\text {video }}=I_{\text {dark }}+I_{\text {stray }}+I_{\text {particles }}
$$

$I_{\text {dark }}$ is measured by switching of the LED illumination and is a constant that can be systematically subtracted from all pixel values. Stray light is relatively strong compared to fluorescence microscopy. $I_{\text {stray }}$ has been measured by injecting buffer solution without particles, and can also be deduced from pixel intensity histogram of videomicroscopy frames of dilute nanoparticle suspension. After subtracting these contributions from the raw video signal, we have the images of the intensity due to the presence of nanoparticles. This gives finally videomicroscopic sequences of electrokinetic behavior of the particles, as illustrated in Figure 5. The background-corrected intensity is - under the proper circumstances - proportional to the nanoparticle concentration, as we previously demonstrated experimentally, ${ }^{21}$ i.e. $I_{\text {particles }} \propto C_{\text {particles }}$ 

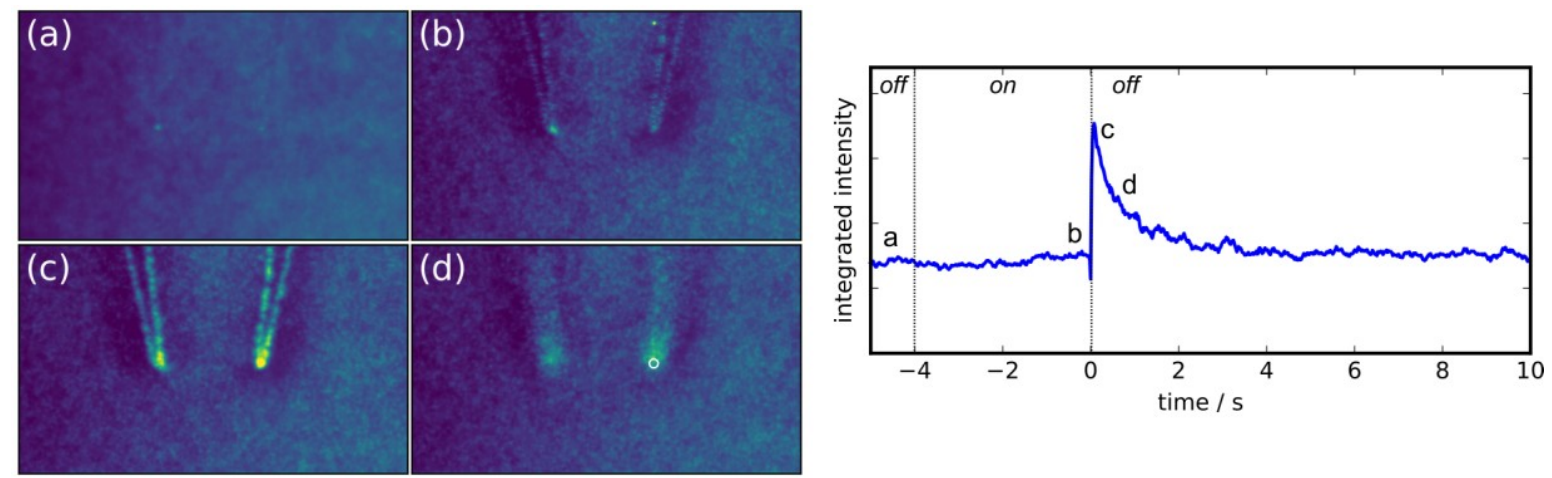

Figure 5. Real-time monitoring of electrokinetic capturing of $150 \mathrm{~nm}$ gold nanospheres using dark-field videomicroscopy, with averaging over several cycles. The video was acquired at $50 \mathrm{fps}$, and several precisely timed 'on-off' cyles were recorded. Left panel: frames of dark-field intensity averaged over several cycles (false color; 'viridis' colormap) at different phases of the cycle: (a) at equilibrium, with E-field switched off $(t=-5 \mathrm{~s})$; (b) after $4 \mathrm{~s}$ of electric field ( $1 \mathrm{MHz}, 10 \mathrm{Vpp})$, just before $t=$ 0 ; (c) just after $\mathrm{t}=0$ when E-field was switched off; (d) at $t=0.9 \mathrm{~s}$, when captured particles have partially diffused away. In (d), the small white circle indicates the zone where the intensity was monitored (area $4.1 \mu \mathrm{m}^{2}$ ). Right panel: intensity vs time in the monitored zone (white circle), averaged over several cycles. The focal plane of observation was close to the electrodes ( $2 \mu \mathrm{m}$ above the electrodes).

During the capturing phase (E-field on, $t=-4 \ldots 0 \mathrm{~s}$ ) there is no significant increase in light intensity in the monitored zone. Particles are being captured, as evidenced by their subsequent release from the capture zone at $t=0$. However, when captured, these particles remain largely invisible. They are optically hidden by the first layer of captured particles. Gold nanoparticles have very large extinction cross sections, and a single layer of particles is sufficient for rendering the zone opaque for further observation.

With more careful observation, and using lower E-field amplitudes, a slight increase of the light intensity from the capturing zone is found. The light intensity rapidly reaches a limiting value. This is shown in Figure 6.
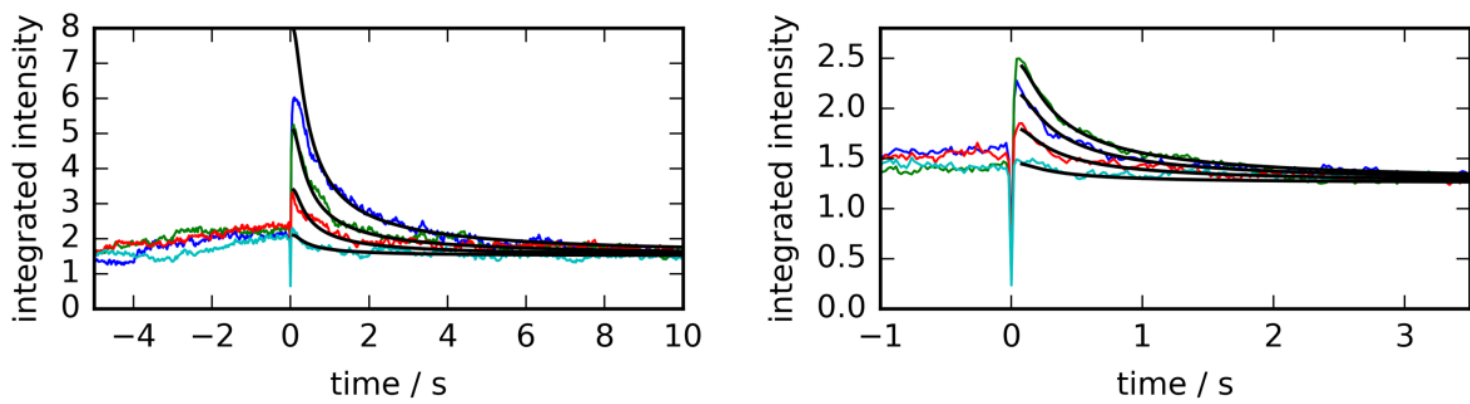

Figure 6. Dielectrophoretic 'capture-release' cycles at different AC field amplitudes (frequency fixed at $1 \mathrm{MHz}$ ), monitored via the integrated intensity in the $4.1 \mu \mathrm{m}^{2}$ circular region-of-interest. The signals are averaged over several cycles (typically between 5 and 15). Top: traces at 15, 10, 5 and $3 \mathrm{Vpp}$. Bottom: traces at 5, 4, 3 and $2 \mathrm{Vpp}$ (zoom for clarity). The colored curves are the experimental traces, the black lines are from a simple theoretical model (see Text) with diffusion coefficient $D$ $=3.3 \mu \mathrm{m}^{2} \mathrm{~s}^{-1}$ and capture radius $a=2.0 \mu \mathrm{m}$.

For analysis of the particle-release curves, we propose here a simple model in which we describe the zone where the nanoparticles are captured as a disk with radius $a$. We consider the release to be followed by a purely diffusional process that we model in $2 \mathrm{D}$. The concentration at the center point of the capturing disk is then given ${ }^{32}$ by Eqn. (3)

$$
C t=C_{e q}+C_{0}\left\{1-\exp \frac{-a^{2}}{4 D t}\right\}
$$


Here, $C_{e q}$ is the equilibrium concentration, $C_{0}$ the observed concentration jump in particles at $t=0, D$ the diffusion coefficient and $a$ the effective radius of the DEP capture disk. We assume that the detected intensity is proportional to the concentration of particles.

In this initial analysis, we use the Stokes-Einstein diffusion coefficient for $150 \mathrm{~nm}$ diameter spheres in water at $\mathrm{T}=298 \mathrm{~K}$ $\left(3.3 \mu \mathrm{m}^{2} \mathrm{~s}^{-1}\right)$, and we adjust the radius $a$ such that the experimental curves are best reproduced. In later work, it will be possible to calibrate $a$ and measure diffusion coefficients of uncharacterised objects using a known particle sample as the reference. As seen in Figure 7, this simple theoretical modeling reproduces the experimentally observed data, even though at higher electric field amplitudes $\left(15 \mathrm{~V}_{\mathrm{pp}}\right)$ deviations begin to appear.

The height of the $t=0$ 'particle release' burst can be assumed to be proportional to the number of captured particles. This height is calculated relative to the equilibrium concentration, i.e. $C_{0} / C_{e q}$. Figure 7 shows the release amplitude obtained for gold nanoparticle samples at various applied AC fields (given as peak-to-peak voltage applied to the electrodes). For low AC fields, the release amplitude varies quadratically as a function of the RMS field.

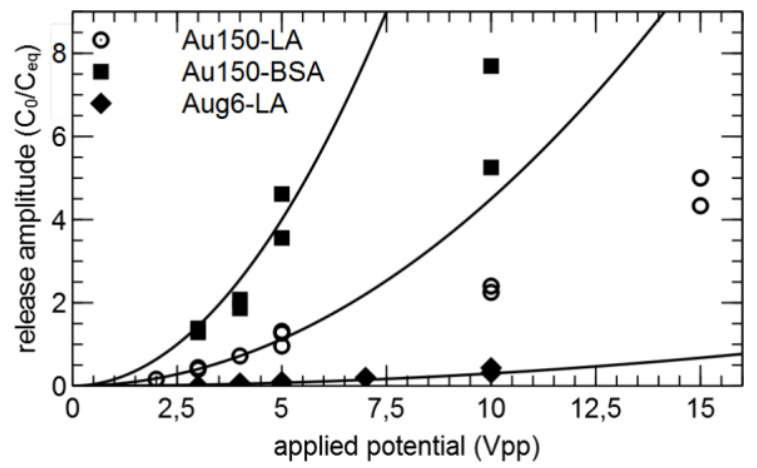

Figure 7. Amplitude of the " $t=0$ " particle release burst as a function of electric field strength. The height of this burst, expressed relative to the equilibrium intensity, is a measure of dielectrophoretic capturing efficiency. The data points indicate individual measurements in different devices. The solid lines are parabolic curves fitted to the experimental data between 0 and $5 \mathrm{~V}_{\mathrm{pp}}$ ).

A parabolic fit of type $y=k x^{2}$ of release amplitude $v s$ applied potential yields a coefficient that represents the dielectrophoretic responsivity of the particular nanoparticle type, and which is proportional to the $r^{3} K_{\mathrm{CM}}$ term in Eqn. (1). We observe that BSA increases the DEP response over three-fold compared to LA for $150 \mathrm{~nm}$ diameter gold particles, indicating that interaction with proteins may enhance DEP at this frequency by changing the Clausius-Mossotti factor $K_{\mathrm{CM}}$. Interestingly, when keeping the surface chemistry constant (Au150-LA vs Aug6-LA) and changing the particle diameter from $150 \mathrm{~nm}$ to $70 \mathrm{~nm}$, the DEP responsivity decreases by a factor of 11, in line with the ratios of the third power of their diameters.

\section{CONCLUSION}

In this work, electrokinectic effects on gold nanoparticle suspensions were characterized in a microfluidic system with integrated planar microelectrodes, using newly developed videomicroscopy under dark-field illumination by LEDs and subsequent image processing. The application of an AC electric field on the colloidal suspensions generated two types of electrokinetic effects: electrohydrodynamic motion of the entire fluid and dielectrophoretic capturing of the nanoparticles at the tips of the electrodes. By working at low E-field amplitude $\left(<10 \mathrm{~V}_{\mathrm{pp}}\right)$, the electrohydrodynamics could be silenced, allowing to study the dielectrophoretic behaviour of the nanoparticles. Bovine serum albumin changes the DEP response of $150 \mathrm{~nm}$ gold nanospheres as compared to lipoic acid, which is a first result towards a more comprehensive study of the effect of biomolecules on the electrokinetics of nanoparticles. Such study can in turn give rise to new in situ characterization methods of nanoparticles in biomedical diagnostic applications. 


\section{ACKNOWLEDGEMENTS}

This work was supported through a Ph.D. fellowship funded jointly by ENS Rennes and Région Bretagne (ARED programme), and through the France-UK Ph.D. programme funded by Dstl. The authors gratefully acknowledge financial support from Agence Nationale de la Recherche (grant ANR-2010-JCJC-1005-1, project COMONSENS) and the Ministry of Science and Technology of Taiwan under the Dragon Gate Project (grant MOST 105-2911-I-007-525).

\section{REFERENCES}

[1] Werts, M. H. V., Allix, F., Francais, O., Frochot, C., Griscom, L., Le Pioufle, B., Loumaigne, M., Midelet, J., "Manipulation and optical detection of colloidal functional plasmonic nanostructures in microfluidic systems," IEEE J. Sel. Top. Quantum Electron. 20(3), 6900613 (2014).

[2] Lafleur, J. P., Senkbeil, S., Jensen, T. G.., Kutter, J. P., "Gold nanoparticle-based optical microfluidic sensors for analysis of environmental pollutants," Lab Chip 12(22), 4651 (2012).

[3] Thiele, M., Soh, J. Z. E., Knauer, A., Malsch, D., Stranik, O., Müller, R., Csáki, A., Henkel, T., Köhler, J. M., et al., "Gold nanocubes - Direct comparison of synthesis approaches reveals the need for a microfluidic synthesis setup for a high reproducibility," Chem. Eng. J. 288, 432-440 (2016).

[4] Knauer, A., Thete, A., Li, S., Romanus, H., Csáki, A., Fritzsche, W., Köhler, J. M., "Au/Ag/Au double shell nanoparticles with narrow size distribution obtained by continuous micro segmented flow synthesis," Chem. Eng. J. 166(3), 1164-1169 (2011).

[5] Werts, M. H. V., Raimbault, V., Texier-Picard, R., Poizat, R., Français, O., Griscom, L.., Navarro, J. R. G., "Quantitative full-colour transmitted light microscopy and dyes for concentration mapping and measurement of diffusion coefficients in microfluidic architectures," Lab Chip 12, 808-820 (2012).

[6] Werts, M. H. V., Raimbault, V., Loumaigne, M., Griscom, L., Français, O., Navarro, J. R. G., Débarre, A.., Le Pioufle, B., "Optical microscopy and spectroscopy of analyte-sensitive functionalized gold nanoparticles in microfluidic systems," Proc. SPIE 8595, 85950W-85950W-11 (2013).

[7] Morgan, H., Green, N. G., AC Electrokinetics: colloids and nanoparticles, Research Studies Press Ltd., Baldock, England (2003).

[8] Nerambourg, N., Praho, R., Werts, M. H. V., Thomas, D., Blanchard-Desce, M., "Hydrophilic monolayerprotected gold nanoparticles and their functionalisation with fluorescent chromophores," Int. J. Nanotechnol. 5(6), 722 (2008).

[9] Loumaigne, M., Praho, R., Nutarelli, D., Werts, M. H. V., Débarre, A., "Fluorescence correlation spectroscopy reveals strong fluorescence quenching of FITC adducts on PEGylated gold nanoparticles in water and the presence of fluorescent aggregates of desorbed thiolate ligands.," Phys. Chem. Chem. Phys. 12(36), 1100411014 (2010).

[10] Saulis, G., Lape, R., Pranevičiute, R., Mickevičius, D., "Changes of the solution pH due to exposure by highvoltage electric pulses," Bioelectrochemistry 67(1), 101-108 (2005).

[11] Jones, T., "Basic theory of dielectrophoresis and electrorotation," IEEE Eng. Med. Biol. Mag. (December), 3342 (2003).

[12] Pethig, R., "Dielectrophoresis: Status of the theory, technology, and applications," Biomicrofluidics 4(2), 022811 (2010).

[13] Zhang, C., Khoshmanesh, K., Mitchell, A.., Kalantar-Zadeh, K., "Dielectrophoresis for manipulation of micro/nano particles in microfluidic systems," Anal. Bioanal. Chem. 396(1), 401-420 (2010).

[14] Honegger, T., Peyrade, D., "Comprehensive analysis of alternating current electrokinetics induced motion of colloidal particles in a three-dimensional microfluidic chip,” J. Appl. Phys. 113(19), 194702 (2013). 
[15] Kayani, A. A., Zhang, C., Khoshmanesh, K., Campbell, J. L., Mitchell, A.., Kalantar-zadeh, K., "Novel tuneable optical elements based on nanoparticle suspensions in microfluidics," Electrophoresis 31(6), 1071-1079 (2010).

[16] Chrimes, A. F., Kayani, A. A., Khoshmanesh, K., Stoddart, P. R., Mulvaney, P., Mitchell, A.., Kalantar-zadeh, K., "Dielectrophoresis-Raman spectroscopy system for analysing suspended nanoparticles.," Lab Chip 11(5), 921-928 (2011).

[17] Loumaigne, M., Midelet, C., Doussineau, T., Dugourd, P., Antoine, R., Stamboul, M., Débarre, A., Werts, M. H. V., "Experimental determination of optical extinction and scattering cross sections of plasmonic nanoparticle dimers in aqueous suspension.," Nanoscale 8, 6555-6570 (2016).

[18] Loumaigne, M., Laurent, G., Werts, M. H. V., Débarre, A., "Photoluminescence spectra and quantum yields of gold nanosphere monomers and dimers in aqueous suspension,” Phys. Chem. Chem. Phys. 18(48), 33264-33273 (2016).

[19] Dominguez-Medina, S., Blankenburg, J., Olson, J., Landes, C. F.., Link, S., “Adsorption of a Protein Monolayer via Hydrophobic Interactions Prevents Nanoparticle Aggregation under Harsh Environmental Conditions.," ACS Sustain. Chem. Eng. 1(7), 833-842 (2013).

[20] Bastús, N. G., Comenge, J., Puntes, V., "Kinetically controlled seeded growth synthesis of citrate-stabilized gold nanoparticles of up to $200 \mathrm{~nm}$ : Size focusing versus ostwald ripening," Langmuir 27(17), 11098-11105 (2011).

[21] Navarro, J. R. G.., Werts, M. H. V., "Resonant light scattering spectroscopy of gold, silver and gold-silver alloy nanoparticles and optical detection in microfluidic channels.," Analyst 138(2), 583-592 (2013).

[22] Shortell, M. P., Hewins, R. A., Fernando, J. F. S., Walden, S. L., Waclawik, E. R., Jaatinen, E. A., "Multi-angle fluorometer technique for the determination of absorption and scattering coefficients of subwavelength nanoparticles," Opt. Express 24(15), 17090 (2016).

[23] Edelstein, A. D., Tsuchida, M. A., Amodaj, N., Pinkard, H., Vale, R. D.., Stuurman, N., “Advanced methods of microscope control using $\mu$ Manager software," J. Biol. Methods 1(2), 1-10 (2014).

[24] Edelstein, A., Amodaj, N., Hoover, K., Vale, R.., Stuurman, N., "Computer control of microscopes using Manager.," Curr. Protoc. Mol. Biol. 14, 20.1-20.17 (2010).

[25] Stuurman, N., “Arduino ( $\mu$ Manager),” $<$ https://micro-manager.org/wiki/Arduino\#Firmware $>$.

[26] Millman, K. J., Aivazis, M., "Python for Scientists and Engineers,” Comput. Sci. Eng. 13(2), 9-12 (2011).

[27] Van Der Walt, S., Colbert, S. C.., Varoquaux, G., “The NumPy array: A structure for efficient numerical computation," Comput. Sci. Eng. 13(2), 22-30 (2011).

[28] Hunter, J. D., "Matplotlib: A 2D graphics environment," Comput. Sci. Eng. 9, 90-95 (2007).

[29] Gohlke, C., "Tifffile (Python)," <http://www.lfd.uci.edu/ gohlke>.

[30] Adrian, R. J., Westerweel, J., Particle Image Velocimetry, Cambridge Aerospace Series (2011).

[31] Thielicke, W., Stamhuis, E. J., "PIVlab - Towards User-friendly, Affordable and Accurate Digital Particle Image Velocimetry in MATLAB," J. Open Res. Softw. 2(1), 30 (2014).

[32] Crank, J., The mathematics of diffusion (2nd Ed.), Clarendon Press, Oxford, UK (1975). 